Aaron J. Butler

\title{
Preliminary Reflections on the Ontological Significance of Blockchain Technology for Trust and Trustworthiness
}

\section{Introduction}

In recent years, there has been a great deal of excitement regarding the promise of blockchain technology (Aste et al. 2017). Yet, not all of this excitement has been positive (Schneier 2019). In fact, the furore raised by the assurance afforded by the capacities of blockchain technology (henceforth: Blockchain) is multifaceted: three such are noteworthy. The first is that Blockchain precipitates a reshaping of our social reality (Reijers \& Coeckelbergh 2018). The second is that Blockchain can be trusted (Aste et al. 2017). The third is that Blockchain can "optimize" trust (Swan \& De Filippi 2017).

For this discussion, I shall focus only on the aforementioned three claims, namely: (1) reshaping our social reality (2) bearing the onus of trust (3) optimization of trust. These three claims clearly have implications for one another. Were Blockchain able to reshape the social reality, then it would thereby reshape trust. This would, in turn, influence the answer to the question: 'Can one trust Blockchain?' This would, likewise, influence an answer to the question, namely: Can Blockchain "optimize" trust?, by the simple fact that it would affect the very nature of the item Blockchain putatively can optimize. The upshot here being: How do these claims form, if at all, a coherent rationally evaluable picture of Blockchain's potential effects in human affairs of great value given, at the very least, the clear implications the first claim has for the latter two?

In this paper, I shall attempt to answer this fundamental question by means of an examination of the associated respective claims. However, in doing so, I shall focus on the first claim and its implications for the other two. What this means is that I shall proceed on the supposition that Blockchain can reshape our social reality. Then, from this supposition, examine how the social ontology of trust and trustworthiness must need be affected. Nonetheless, I shall, through the course of the examination, also evaluate the trust-based claims of Blockchain: as to its trustworthiness and ability to optimize both trust and trustworthiness. 
Now, to this approach, one might dissent noting that my analysis would be limited by its suppositional structure such that the answer wrought would be changed were the supposition not granted. I reply that there is an integral relationship between the reshaping of our social reality, and thereby trust and trustworthiness, such that the virtues Blockchain is presumed to have are a result of this very reshaping. Hence, my analysis, although limited in scope: to be sure, is not flawed as a result, just modest and wedded to the mainstay conceptual connection between a Blockchainmodified-social-reality - or at least claims to this effect - and the reshaping of trust and trustworthiness (more on that to follow); or so I hope to show.

The structure of the discussion is as follows. First, I shall explicate the target items of analysis, namely Blockchain's ability to: (1) reshape our social reality (2) bear the onus of trust (3) optimize trust; in so doing, I hope to elaborate further as to the rational motivation of this discussion. Second, I shall articulate in what sense 'social ontology' is being used within the parameters of this discussion and provide an articulation as to what Blockchain is and how it affects our social reality in the sense relevant for this discussion. Third, I articulate the social ontological features regarding trust and trustworthiness that are relevant for this discussion and investigate some of the relevant implications for trust and trustworthiness the influence of Blockchain provides. I conclude with final reflections.

\section{Rational Motivation}

Blockchain is perceived as not just another innovative digital technology within the digital age (i.e., the $4^{\text {th }}$ Industrial Revolution), but rather as a signpost indicating the future of the age of digitalization (Polrot 2017, $1-10$ ), all of which suggests a reshaping of our social reality (Reijers \& Coeckelbergh 2018). The perception that Blockchain can reshape our social reality is precipitated by the understanding that such technology can "transform organizations, democratic governance and human culture as a whole" (Reijers \& Coeckelbergh 2018, 104; Tapscott \& Tapscott 2016). But given Blockchain's putative wide-sweeping powers to reshape our social reality, it becomes pivotal to understand the implications this state of affairs would pose for trust and trustworthiness. Thereupon, we need to be clear on the implications Blockchain has for trust and trustworthiness. This is because having a reflective and discerning understanding of our social reality bodes to improve our understanding of how Blockchain affects that social reality; this, in turn, bodes not only to improve our understanding of the relevant 
effects in question, but it also promises to steer usage along a course more beneficial to human flourishing, as well as holds out promise to shore up our vigilance to have "machines treat us well" (Moor 2006, 21).

As such, can one trust Blockchain? There are a growing number of experts and enthusiasts who would endorse Blockchain thereby providing a positive answer to the question (Woolf 2018; Polrot 2017, 1-10; Reijers $\&$ Coeckelbergh 2018). Some even say that Blockchain can better ward of corruption and improve transparency at both personal and institutional levels (Ishmaev 2017), thus creating an environment more conducive to promoting and maintaining trust, and thereby in effect "optimizing" trust (Kshetri 2018). Still others are skeptical: thinking, among other things, that trust-based assertions regarding Blockchain are grandiloquent or at the very least overstated (Schneier 2019).

Blockchain, as a digital technology, was initially utilized within the financial industry; and as such, it functioned as a means of securing financial transactions involving cryptocurrencies (Dos Santos 2017; Reijers \& Coeckelbergh 2018; Coeckelbergh, et al. 2018). In light of its outstanding success in this regard, there was motivation to apply it to other areas of human affairs: (1) politics (Velasco 2017; Bhaskar 2017, 1-9; Crichton 2018) (2) institutional property management (Ishmaev 2017) (3) smart contracts and Internet of Things (IoT) applications (Christidis \& Devetsikiotis 2016); to name just a few additional applications outside of the financial sector. The appeal of these extra-financial-sector applications is that Blockchain is perceived as being able to mitigate the negative effects (or ideally: doff the negative effects) of moral corruption, aid in upholding the integrity of relevant current relationships obtaining between relevant parties and items (e.g., the seller, the item sold, and the buyer or the voter, the vested-political interest, and the political party or the contractor, the contract, the item contracted, and the contractee), bolstering fairness and fair play. Moreover, Blockchain is perceived to be efficacious in this regard due to its design features. It is precisely these system features, and the system taken as a whole, that affords Blockchain the capacity to shore up trust by means of creating a more reliable environment for imperfect trustors or non-trustors and shifting the onus probandi of/for trust from the relevant ethical actors to the system itself. That is, Blockchain allows for the optimization of the conditions conducive to trust-based interactions, some of the details of which are to follow through the course of this discussion.

In light of the aforementioned, two central issues, namely: (1) 'Can one trust Blockchain?' (2) 'Can Blockchain optimize trust?', needing-to-beaddressed come into sharper focus. This is because an answer to these 
two questions pivotally affects utilization of Blockchain in any domain of human affairs for a rather intuitive reason: If it cannot be trusted or its ability to improve trust is overplayed, what would be the rational motivation for continued usage of it? Hence, these issues have direct relevance for the rationality of praxis, given the domains in which utilization of Blockchain is perceived to be worthwhile or efficacious. Herein, I attempt to address these issues by means of, at the very least, a discussion of some of the conceptual requirements of what a cogent answer to each of these interrogatives would need to satisfy. In so doing, I hope to clear up some conceptual matters that I, in turn, hope will function as part of the hermeneutical framework with which to make sense of the cogency of certain trust-based claims extolling the virtues of Blockchain. Admittedly, to fully evaluate every trust-based claim about Blockchain, given their respective domains of inquiry would be beyond the scope of the current discussion requiring a more fine-grained analysis that simultaneously is wider in scope and wedded to the particular details of the relevant domain of inquiry within which the technology is employed - "the devil is in the details", as the old adage goes. I, rather, aim at a more modest analysis that is, nevertheless, grounded in first principles and capable of investigating the fundamental conceptual features common to trust-based applications of Blockchain across a wide variety of domains within which the technology is applied.

There are several facets to our social reality: ontological, epistemological, axiology, etc. The focus of the current discussion is the ontological aspects of social phenomena. For the best way to evaluate the coherence of the relevant claims regarding Blockchain is to examine the ontological aspects. This is so due to the nature and properties of the social entities that Blockchain is supposed to be able to influence. So, the most direct way to evaluate the coherence of the aforementioned claims is a direct examination of the social ontological aspects of our reality putatively affected.

We shall now take a direct look at some of the relevant issues surrounding Blockchain and our social reality with peculiar attention to examine the issue of the reshaping of the social reality of trust and trustworthiness. The added benefit of this way of approaching the issues in question is that it allows for the opportunity to examine some of the relevant conceptual features concomitant with Blockchain's influence on our social reality. 


\section{Social Ontology, Blockchain, Trust $\&$ Trustworthiness}

\subsection{Social Ontology}

Social ontology is the investigation into the "nature and properties of the social world" (Epstein 2018). Ultimately, its mainstay concern is with the analysis of multifarious "entities" arising out of "social interactions" (Ibid, 1). Some such notable items being currency, property, institutions, corporations, class, race, gender, artefacts, language, public policy, and law. Moreover, there is a great deal of overlap between philosophical and social scientific investigations into these items. Nevertheless, the philosophical pursuits also tend, treating the field of social ontology as a subfield of metaphysics: the latter being understood as "the general inquiry into the nature of entities", to concern itself with the very nature of social reality: its basic elements, building blocks, development, and transformations (Ibid, 1; Searle 1996, 2010).

It is along these lines that the present philosophical investigation is to be understood, namely: as an inquiry into the nature of Blockchain`s transformative effect (if any) on the social entities of trust and trustworthiness. This investigation yielding, or so it is hoped, a better understanding as to whether or how the social ontology of trust and trustworthiness are changed as a result. Now, some might object that this investigation is not something for which philosophy is competent to engage. This is because the relevant investigation is an empirical matter.

Admittedly, this investigation is, to some extent, empirical, but not without philosophical consequence(s). Moreover, it is the philosophical consequence(s) that primarily concern this paper, but not to the extent of insensitivity to the value of the empirical component. Empirical investigation, however, is insufficient to address the pertinent issues herein. For the question is concerning the nature and properties of social entities. Observation, however sophisticated, cannot, for example, explain or elucidate what a promise is. Sure. Empirical investigation can help better understand the practice of promise-making, but not what a promise is when one is being made nor can empirical investigation elucidate certain other quandaries, such as what social entities' individuation conditions, persistence conditions, sortal-membership, parthood (if any), and the like are (Wiggins 2001; Gallois 2016; Varzi 2016). No. These quandaries require philosophical investigation to be pursued well, since philosophical investigation utilizes the rigors of conceptual analysis as a mainstay tool, and 
it is conceptual analysis which is fit for the task. For conceptual analysis allows one better to understand the underlying principles responsible for the nature and properties of social entities as well as the abstract properties and relations that social entities have or into which they enter. Empirical investigation is not amenable to elucidating such things. Conceptual analysis also allows us to develop clear and dynamic representational models of the behavior of social entities in a given social environment.

One might rejoin that empirical investigation involves conceptual analysis. Thus, empirical investigation is sufficient to answer the relevant questions and amenable to elucidating the nature and properties of social entities. But I reply that to a certain extent this is so, but not to the extent to be reliable so as to understand the intangible features of social entities, like for example, the nature of a promise or the abstract relationships that must obtain for the existence of an institution. As such, certain inquiries into social entities or the social space require a philosophical investigation into the ontology of the relevant social entities under consideration: an empirical investigation cannot do this regardless of whether it involves, to a certain extent, conceptual analysis.

We now turn to a fuller articulation as to what Blockchain is and how it relates to philosophical inquiries into social ontology.

\subsection{Blockchain}

Blockchain is a decentralized digital ledger technology conforming to the following criteria:

(1) Being a database

(2) Using encryption protocols and algorithms

(3) Being decentralized and distributed among participating members of a network

(4) Being transparent, trackable, and publicly accessible

(5) Having validation parameters for acceptable inputs and a mechanism of consensus tracking to ward off tampering

all of which afford for secure transfer of money, information, and digital assets using encryption protocols and algorithms in such a way that a permanent record of all transactions is securely stored and publicly vouchsafed by the system itself. This is, in fact, wherein its power lies: (1) security, (2) immutability (3) transparency, taking the place of third-party personal or institutional trust safeguards (Swan \& De Filippi 2017, 603-604). All of these features and capacities of Blockchain poise the technology so that it 
can take on the onus probandi of trust, in effect taking it out of the hands of the social-world actor; thus, transforming the very conception of trust (more on this to follow) and, at the very least, putatively optimizing the very conditions conducive to attributing and grounding trustworthiness and facilitating the development of trust on a sturdier basis.

One reason why the aforementioned, namely: poising Blockchain to take on the onus probandi of trust and "optimize" trust, is even the case is that Blockchain provides a way to reproduce the opportunities of trust that obtain between two individuals, meeting in person, to exchange goods or services. This being enabled by system features possessed of Blockchain such that digital representations (second-order level) of relevant services or material items (first-order level) are given characteristics not unlike services and material items in the sphere of action (Reijers \& Coeckelbergh 2018). Thereupon, digital items are put on par with material goods and human action.

Now, the aforementioned transformation of social organizations, institutions, and the like precipitated by Blockchain (Reijers \& Coeckelbergh 2018), along with the repositioning of the status of digital representations (again: second-order) and their corresponding actual objects (again: firstorder) bespeak of Blockchain's power to reshape our social ontology, putatively, affecting both trust and trustworthiness. But as to the nature of trust and trustworthiness such that they can even be affected by Blockchain, this follows forthwith.

\subsection{Trust \& Trustworthiness}

There are many aspects to the philosophy of trust: for this discussion, the very nature of trust is relevant (McLeod 2015). Received philosophical opinion holds that trust is an attitude and trustworthiness a property (Ibid). The former being an attitude poses no special problems for social ontology, as one major concern for the latter just is how one bridges an analysis of psychological states, on the one hand, and social phenomena, on the other hand (Epstein 2018). Nonetheless, the sortal of the relevant items is important (Wiggins 2001), because it is important to understand the sort of items under investigation in order to, in turn, better understand how such items interact with Blockchain.

Other than the relevant sortals each item - trust and trustworthiness falls under, there are a few other features of the relevant items to bear in mind. As such, there are two paradigms of trust. The traditionally major 
one is that of interpersonal trust; that is, trust is something that obtains between persons, and whence is enriched and extended producing other variants that are, thereby, parasitic on the main conception (McLeod 2015). Consonant with this paradigm is the "contractarian-individualist" view on trust (Coeckelbergh 2012, 54), which holds that trust is an attempt to make a decision in the face of uncertainty as to the outcome and vulnerability, on the part of the trustor, to betrayal: risk assessment on the part of the trustor in relation to the trustee regarding some action, set of actions, or circumstances (Taddeo 2009; Coeckelbergh 2012; McLeod 2015). The minor paradigm holds that trust is a given, a part of one's naive ontological attitude regarding one's comportment in and relationship to the social world (Coeckelbergh 2012).

Furthermore, there is no rational basis for trust in an outcome that is guaranteed: under such circumstances trust would, indeed, be irrelevant (McLeod 2015). Additionally, some philosophers would further distinguish between trust and reliance holding that reliance can exist without the attitude of trusting: one would then, should things not work out, be disappointed, yet not betrayed. Moreover, for someone to have the property of trustworthiness, that one must also have the power to betray us (Holton 1994). Still other philosophers acknowledge the possibility of trust as reliance, as in the case of trusting artefacts (Coeckelbergh 2012). Thus, blurring the traditional lines between trust and reliance, as well as shifting what grounds trustworthiness: the power to betray no longer being relevant here, except for possibly in cases of advanced artificial intelligence, artificial general intelligence, and super intelligence (Bostrom 2012; Bostrom \& Yudkowsky 2014, 316-334; Bostrom 2017, 12-17); but rather reliability as an empirical matter (Reijers \& Coeckelbergh 2018).

\section{Critical Reflections}

If Blockchain can "transform organizations, democratic governance and human culture as a whole" (Reijers \& Coeckelbergh 2018; Tapscott \& Tapscott 2016), then it can reshape our social reality only if it reshapes other key aspects of our social reality. For example, an organization attempts to use Blockchain. As such, they are condoning the use of Blockchain as a substitute for roles traditionally filled by human agents. Insofar as they do, they are evidencing a different understanding of what is enough (or even what is necessary) to fill a social role. The upshot of this is that for the claim that Blockchain can shape our social reality to be true, extant instances of 
Blockchain affecting various aspects of our social reality is enough. Along these lines, if Blockchain can transform our social reality thusly and trust and trustworthiness are some of these aforementioned key aspects, then Blockchain can reshape trust and trustworthiness. Yet, it is important to note that the reasoning, prima facie, doesn't go through. For it does not follow that simply because Blockchain affects various aspects of our social reality, it thereby affects trust and trustworthiness in particular.

Nevertheless, there is a substantive sense in which the effect that Blockhain has on various aspects of our social reality does thereby affect trust and trustworthiness. This is because for Blockchain to generally have this effect, its influence would have to go beyond exceptional cases affecting the fundamental social entities that are necessary for having organizations, institutions, and governments: trust and trustworthiness. Accordingly, there is an important logical relationship here, namely: one of sufficiency such that were Blockchain able to reshape our social ontology, then it would thereby reshape the social ontology of trust and trustworthiness. Consequently, there is an integral conceptual connection between the reshaping of our social ontology and the reshaping of the social ontology of trust and trustworthiness such that a change in the former would precipitate a change in the latter. So, in order to better understand all of this, it is useful simply to grant that Blockchain can indeed reshape in the sense relevant for this discussion and then explore the impact, if any, of this for the social ontology of trust and trustworthiness.

So, suppose Blockchain can reshape or transform our social reality (i.e., social ontology) thusly. Then, it would change social entities therein; this involves, at least, reshaping our understanding of such entities, or at most, reshaping our practices regarding such entities. Trust and trustworthiness are instances of the relevant type of social entities. Blockchain, then, reshapes trustworthiness by substituting for trust-based intermediaries; thus, precipitating a change in the onus of trust from that of social actors to the system itself (as has been discussed above) (Swan \& De Filippi 2017, 603604). This, in turn, would change our conception of social or interpersonal trust (major paradigm for the contemporary conception of trust; 'social trust', here, being used synonymously with 'interpersonal trust') from that which obtains between persons to that which obtains between human persons and the instrumentality of an artefact or tool. Yet, it is not true that Blockchain is a substitution for trust-based intermediaries, and this being so by means of its own design parameters. For if something were to go awry with some Blockchain facilitated activity, who would be turned to for recourse? Some system engineer, legal counsel, or form of governance 
- a trust-based intermediary to be sure. Thence, our conception, and even practice of trust, would not be changed: it is still grounded, at least in part, in an interpersonal dynamic. Thereupon, Blockchain cannot reshape our social reality (i.e., social ontology) in the sense relevant for this discussion.

Some might inveigh that the claim, namely: yet, it is not true that Blockchain is a substitution for trust-based intermediaries, and this being so by means of its own design parameters, is a result deduced from the reasoning thus far. But this is absolutely not the case. For the claim expresses the realization that the technology's own design parameters require involvement of trust-based intermediaries. Nonetheless, one might say that the result is a trivial one that comes through the course of a much belabored argument. This is because this result could have been elaborated first so as to avoid protracted argumentation beyond what is really necessary. The upshot of this objection being: the argumentation in question suffers from triviality and violates the principle of parsimony, respectively, in that: (1) the step in the argument expresses a basic design feature of the technology doing so in such a way that (2) the argument expands unnecessarily occluding key factors best to be mentioned earlier in the argument on pain of cumbersomeness and verbosity. On the contrary, the result is not trivial. Simply because the design feature in question, namely: that Blockchain requires involvement of trust-based intermediaries, is basic doesn't mean it is trivial. Some basic features are significant due precisely to being basic. This is one such feature, which substantiates the fact that it is fundamental to Blockchain's functionality as the system that it is. Moreover, the argument doesn't violate parsimony or if it does, it would not do so anymore than any other argument of the form reductio ad absurdum. Furthermore, one of the benefits of the current structure of the discussion thus far is, or so I hope, that the provisional assumption, which kicks off the argument in question, provides a clearly articulated rational motivation as to why one would assert such a claim regarding Blockchain. Hence, the provisional assumption, or so I have attempted to show, represents a claim possessed of a non-trivial degree of prima facie cogency. The upshot here being, namely: refraining from believing that a view in question has little in the way of a cogent rational motivation for asserting or even entertaining whether it is true. Additionally, there are benefits to proceeding argumentatively in this manner. The first is the aforementioned benefit of optimization of the cogency of the claim in question. The second is that if it can be shown that the claim, despite having optimized cogency, is nonetheless false, then all the better for the critique of it. 
Whilst fully supporting the argument against the coherence of the claim, namely: that Blockchain can reshape our social reality, I note the argument's soundness is restricted by certain hermeneutical limits. Truth is always under an interpretation, and an argument is sound if it is valid and has all true premises. Thus, the truth of each step of the justification portion of the argument is restricted by certain hermeneutical limits. But what are these limits? Well, the argument is not supporting the claim that all across the board Blockchain cannot reshape our social reality. For there are clear counterexamples to this: liquid democracy (Crichton 2018). It is also not saying there are no bona fide instances of a trust-based intermediary being substituted; for there are, indeed, cases of these and the theoretical basis to support them (Reijers \& Coeckelbergh 2018; Coeckelbergh, et al. 2018; Nakamoto 2008).

But Blockchain's effect on trust is not quite right: it cannot bear the onus of trust, because it still shares this burden with human actors (e.g., computer system engineers, legal counsel and governance officials), nor can it substitute for trust-based intermediaries across the board. Moreover, it does not "optimize" trust in the sense that it makes conditions of trustworthiness better because it doesn't entirely eliminate the messiness of involvement of the "hands" of trust-based intermediaries. Furthermore, it does not reduce the category of interpersonal-based trust to a species of trust as reliance (as is, arguably, the case with tools) due to the fact that there are still human agents one turns to for recourse, should something be amiss.

Yet, there is a sense in which Blockchain does bear the onus of trust, as co-bearer of the onus of trust, similar to a roller coaster: a pre-digital age technology. The automation it represents, its structure, et cetera, in a nontrivial sense bear the burden of trust, along with the engineers, financiers, policymakers and the like. Would this similarity not call into question the claim to Blockchain's uniqueness, especially with respect to the digital age?

Nonetheless, looking at the optimization-claim from a different perspective, nay, a different model, shows that optimization of trust is not entirely a wild claim with little merit. Perhaps a signal-detection-based model might prove useful in making the point clear (Wickens 2001). Recall that this model serves as a way of representing a system (usually physical in nature) of interest with respect to some information-imparting feature of the system amidst other information-imparting features. As such, the model represents the system in terms of a ratio of signal to noise. So, when one has a lot of confounds - noise - then the target item - signal - is going to be harder to detect, and vice versa. Analogously, focusing on the ratio of noise to signal, reducing the noise in order to maximize detection of the signal, 
social or interpersonal trust is a messy business: there is risk of betrayal, information deficits, concerns regarding sincerity and motives and other relevant psychological traits, the need for confirmation (in some cases), and the difficulties of knowing whether that confirmation is genuine (or just an "echo" of what the trustor wished to "hear").

Blockchain bodes to streamline all of this by attempting to reduce relevant categories of trust (i.e., interpersonal trust) to trust as reliance. Then, loading the burden of these other categories of trusting, their satisfaction conditions, requirements, et cetera, onto the Blockchain (or the practice of using the Blockchain) system, thereby narrowing our performance surrounding trusting and evaluating trustworthiness. Additionally, on this model, the performance aspects of trust are being reshaped and trust is being optimized. This, of course, is on the assumption that the signal detection model is the correct model with which to base one's explanatory and predictive accounts of the social ontological relevance of trust and trustworthiness, given the use and influence of Blockchain: an assumption for which justifying it falls outside the purview of this discussion; nonetheless illustrating this possible model's explanatory merits is beneficial, since it facilitates conceptual analysis aimed at evaluating the cogency of claims made regarding Blockchain.

\section{Conclusion}

Herein, I have attempted to show that Blockchain, though able to reshape our social ontology, the claim having been granted, has limited effects on the social ontology of trust and trustworthiness. This, however, does not rule out Blockchain's ability to reshape other aspects of our social ontology.

I have also attempted to address two intuitively compelling auxiliary interrogatives, namely: (1) 'How do the three mainstay interrogatives (see introduction) form, if at all, a coherent rationally evaluable picture of Blockchain's potential effects in human affairs of great value given, at the very least, the clear implications the first claim has for the latter two?' (2) 'If it cannot be trusted or its ability to improve trust is overplayed, what would be the rational motivation for continued usage of it?'. To the first auxiliary interrogative, I answer that they do not; that is, they don't form a coherent rationally evaluable picture, unless put within the hermeneutical matrix limiting the scope and fecundity of Blockchain for our social reality. 
To the second auxiliary interrogative, Blockchain is, clearly, still useful and our understanding of the technology is still developing; thus, our understanding of its effects on our social reality is also likely still growing. Overtime, as praxis becomes more secure, our understanding bodes to brighten as well. This promises not only to aid in deepening our understanding of our social reality, but the very nature of philosophy, and thereby the philosophy of technology itself. Whence a metaphilosophical perspectivea view from above - resides, with time, perhaps greater clarity can be garnered.

\section{Bibliography}

Aste, Tomaso, Paolo Tasca, and Tiziana Di Matteo. 2017. "Blockchain Technologies: The Foreseeable Impact on Society and Industry." Computer 50 (9) 18-28.

Bhaskar, Shiva. 2017. "How Blockchain Can Improve Politics." Medium, 31 July 2017. https://medium.com/@simon-polrot/blockchain-state-of-the -art-and-prospective-4777e329df14.

Bostrom, Nick. 2012. "The Superintelligent Will: Motivation and Instrumental Rationality in Advanced Artificial Agents", Minds \& Machines, 22: 71. https://doi.org/10.1007/s11023-012-9281-3.

Bostrom, Nick and Yudkowsky, Eliezer. 2014. "The ethics of artificial intelligence", in William M. (eds.) The Cambridge Handbook of Artificial Intelligence. Cambridge: Cambridge University Press, Cambridge.

Bostrom, Nick. 2017. "The ethical issues of advanced artificial intelligence." Paper presented at the IIAS 2003, Baden Baden, DE. In Smit, S., Wallach, W., and Lasker, L. (eds.) Cognitive, Emotive and Ethical Aspects of Decision Making in Humans and in Artificial Intelligence, vol. 11, IIAS, 12-17.

Christidis, Konstantinos and Devetsikiotis, Michael. 2016. "Blockchain and Smart Contracts for the Internet of Things.” IEEE 4: 2292-2303.

Coeckelbergh, Mark. 2012. "Can We Trust Robot?" Ethics and Information Technology 31: 9-14.

Coeckelbergh, Mark; DuPont, Quinn; Reijers, Wessel. 2018. "Towards a Philosophy of Financial Technologie." Philosophy \& Technology 31: 9-14. Crichton, Danny. 2018. "Liquid democracy uses blockchain to fix politics, and now you can vote for it." Tech Church, 24 February, 2018. https://tec hcrunch.com/2018/02/24/liquid-democracy-uses-blockchain/ 
Dos Santos, Renato. 2017. "On the Philosophy of Bitcoin/Blockchain Technology: Is It a Chaotic, Complex System?” Metaphilosophy Symposium 48 (5): 620-633.

Epstein, Brian. 2018. "Social Ontology." Stanford Encyclopedia of Philosophy, Metaphysics Research Lab, Center for the Study of Language and Information (CSLI), Stanford University, 21 March 2017.

https://plato.stanford.edu/entries/social-ontology/.

Gallois, Andre. 2016. "Identity Over Time." Stanford Encyclopedia of Philosophy, Metaphysics Research Lab, Center for the Study of Language and Information (CSLI), Stanford University, 06 October 2016.

https://plato.stanford.edu/entries/identity-time/.

Holton, Richard. 1994. "Deciding to Trust, Coming to Believe." Australasian Journal of Philosophy 86(2): 213-236.

Ishmaev, Georgy. 2017. "Blockchain Technology as an Institution of Property." Metaphilosophy Symposium 48(5): 666-686.

Kshetri, Nir. 2018. "Using Blockchain to Secure the "Internet of Things"." Electronics, Scientific American, 10 March 2018, accessed 30 April 2019.

McLeod, Carolyn. 2015. "Trust.” Stanford Encyclopedia of Philosophy, Metaphysics Research Lab, Center for the Study of Language and Information (CSLI), Stanford University, 03 August 2015.

https://plato.stanford.edu/entries/trust/.

Moor, James H. 2006. "The Nature, Importance, and Difficulty of Machine Ethics." IEEE Intelligent Systems 21(4): 18-21.

Nakamoto, Satoshi. 2008. "Bitcoin: A Peer-to-Peer Electronic Cash System.” (Whitepaper). No publisher.

Polrot, Simon. 2017. “"Blockchain": State of the Art and Prospective." Medium, 31 January 2017.

https://medium.com/@si-monpolrot/blockchain-state-of-the-art-and-prosp ective-4777e329df14.

Reijers, Wessel and Coeckelbergh, Mark. 2018. "The Blockchain as a Narrative Technology: Investigating the Social Ontology and Normative Configurations of Cryptocurrencies." Philosophy \& Technology 31: 103130.

Searle, John R. 1996. The Construction of Social Reality. London: Penguin Group.

Searle, John.2010. Making the Social World: The Structure of Human Civilization. New York: Oxford University Press.

Schneier, Bruce. 2019. "There's No Good Reason to Trust Blockchain Technology.” Wired, February 6, 2019. 
https://www.wired.com/story/theres-no-good-reason-to-trust-blockchain-te chnology/.

Swan, Melanie and De Filippi, Primavera. 2017. "Toward A Philosophy of Blockchain: A Symposium Introduction.” Metaphilosophy Symposium 48 (5): 603-619.

Taddeo, Mariarosaria. 2009. "Defining Trust and E-Trust: From Old Theories to New Problems." International Journal of Technology and Human Interaction 5(2): 23-35.

Tapscott, Don and Tapscott, Alex. 2016. Blockchain Revolution: How the Technology Behind Bitcoin is Changing Money, Business and the World. New York: Penguin Random House.

Varzi, Achille. 2016. "Mereology." Stanford Encyclopedia of Philosophy, Metaphysics Research Lab, Center for the Study of Language and Information (CSLI), Stanford University, 13 October 2016.

https://plato.stanford.edu/entries/mereology/.

Velasco, Pablo R. 2017. "Computing Legers and the Political Ontology of the Blockchain.” Metaphilosophy Symposium 48 (5): 712-726.

Wickens, Thomas D. 2001. Elementary Signal Detection. Oxford: Oxford University Press.

Wiggins, David. 2001. Sameness and Substance Renewed. Cambridge: Cambridge University Press.

Woolf, Nicky. 2018. "What Could Blockchain Do for Politics?.” Medium, 8 January 2018. https://me-dium.com/@NickyWoolf. 
